\title{
Psicooncología
}

ISSN: 1696-7240

http://dx.doi.org/10.5209/PSIC.57090

\section{Barreras percibidas por el personal de salud para la toma de la citología cervical en mujeres zapotecas de Juchitán, Oaxaca}

\author{
Minerva Saldaña-Téllez ${ }^{1, *}$; María Montero y López Lena
}

Recibido: 15 de mayo de 2017 / Aceptado: 25 de agosto de 2017

Resumen. Antecedentes: El cáncer cervicouterino $(\mathrm{CaCU})$ representa un problema de salud pública en México. Las mujeres indígenas presentan mayor riesgo de morir por esta enfermedad. El estudio sobre las barreras que afronta la población indígena mexicana para la toma de la citología cervical es escaso. Propósitos: Objetivo: Identificar las barreras estructurales, psicosociales y culturales percibidas por el personal de salud para la toma de la citología cervical en mujeres juchitecas. Método: Tipo de estudio: exploratorio-descriptivo de corte cualitativo. Se aplicaron entrevistas semiestructuradas a personal sanitario de centros de salud públicos y privados de Juchitán. Muestreo intencional por cuotas, 9 participantes, 7 clínicas elegidas al azar. Se realizó análisis de contenido. Resultados: Se identificaron barreras estructurales, psicosociales y culturales. Las principales: tardanza en entrega de resultados, falta de material, espacios para la toma y recursos humanos, alto nivel de desinformación sobre la prueba y la enfermedad, miedo a tener algo "malo" y al dolor, el tabú de la sexualidad, la actitud de la pareja hacia la prueba, prejuicios y falta de traductores que apoyen en la comunicación entre las mujeres y el personal del sistema de salud. Conclusiones: Existe desinformación sobre el $\mathrm{CaCu}$ y su detección oportuna en esta población; hay múltiples barreras de tipo estructural, prejuicios y mitos rodean la enfermedad. Se detectó desinterés por parte del sistema de salud en la promoción de programas preventivos del $\mathrm{CaCu}$. Informar a la población en su lengua, cuidar y ser empáticos ante la exposición del cuerpo y diseñar políticas de salud incluyentes, son aspectos fundamentales a considerar y desarrollar en programas locales.

Palabras clave: Cáncer cervicouterino, detección oportuna, mujeres indígenas, desigualdades en salud

\section{[en] Perceived barriers by health care professionals to cervical cancer screening in indigenous women of Juchitan, Oaxaca}

\footnotetext{
Abstract. Background: Cervical cancer is a public health problem in Mexico. Rural and Indigenous women have a higher risk of dying from this disease. The study of the barriers to cervical cancer screening in indigenous population in Mexico is limited. Purpose: Objective: To identify structural, psychosocial and cultural barriers perceived by health care professionals to cervical cancer screening in indigenous women of Juchitán. Method: Exploratory-descriptive study with qualitative approach. Semi-structured interviews with health care professionals in public and private centers in Juchitán were conducted. Intentional sampling by quotas was used. There were 9 participants in 7 randomly selected clinics. Content analysis was performed. Results: Structural, psychosocial and cultural barriers were identified. Delay in delivery of results, lack of materials, specific spaces

1 Universidad Nacional Autónoma de México (UNAM), México.

2 Universidad Nacional Autónoma de México (UNAM), México.

E-mail:monterol@unam.mx

* Dirección de correspondencia: Facultad de Psicología-UNAM. División de estudios de Posgrado. Av. Universidad 3004, Edif. "D” 2do. piso cubículo 7. colonia: Copilco Universidad. México, D.F. 04510 E-mail: mimba007@hotmail.com / psicologaminerva@gmail.com.
} 
and human resources, misinformation, fear of receiving "bad news" and fear of pain, sexuality taboos, partner's attitudes related to the test, prejudices and lack of translators who support the communication between the women and the personnel in the health system, were the most salient barriers to screening. Conclusions: There is misinformation about cervical cancer and screening in this population; prejudices and myths about the disease were reported. There was a lack of interest on the part of health workers in the promotion of preventive cervical cancer programs, even showing discriminatory behavior. Informing the population in their language, caring and being empathetic before the body>s exposure and designing inclusive health policies are fundamental aspects to consider and develop in local programs.

Keywords: Cervical cancer, screening, indigenous women, health imparities.

Sumario. 1. Introducción 2. Participantes y Métodos 3. Resultados 4. Discusión 5. Conclusiones 6. Referencias bibliográficas.

Cómo citar: Saldaña Téllez M, Montero y López Lena M. Barreras percibidas por el personal de salud para la toma de la citología cervical en mujeres zapotecas de Juchitán, Oaxaca. Psicooncología 2017;14:343-364. Doi: 10.5209/PSIC.57090.

\section{Introducción}

Aunque existen avances en el diagnóstico y tratamiento del cáncer cervicouterino $(\mathrm{CaCu})$, éste representa la cuarta causa de mortalidad femenina en el mundo. En el año 2012, hubo 528.000 nuevos casos y 266.000 muertes por esta enfermedad ${ }^{(1)}$. En México, en el año 2008, se registraron 10.186 nuevos casos $^{(2)}$ y en el año 2012 fallecieron 3.840 mujeres mexicanas por $\mathrm{CaCu}$ (Instituto Nacional de Estadística y Geografia ${ }^{(3)}$.

No obstante, este tipo de cáncer tiene una alta probabilidad de curación si se detecta oportunamente. En países desarrollados, se ha visto que la disminución de la mortalidad por $\mathrm{CaCu}$ en mujeres, se debe principalmente a la detección y el tratamiento oportuno de lesiones precursoras y malignas a través de los programas de detección oportuna del $\mathrm{CaCu}^{(4)}$. La toma de la citología cervical o Papanicolau (Pap) es el método de detección oportuna a nivel mundial ${ }^{(5)}$.

En México, los primeros programas encaminados a la detección oportuna del $\mathrm{CaCu}$ se crearon hace aproximadamente 40 años, sin embargo, -a diferencia de algunos países desarrollados- la mortalidad por esta enfermedad no ha disminuido como se esperaba. En el año 1974 arrancó el Programa Nacional de Detección Oportuna de Cáncer (PNDOC), no obstante, la tasa de mortalidad por $\mathrm{CaCu}$ durante los 25 años siguientes no disminuyó debido a la baja cobertura y a los bajos estándares de calidad $^{(6)}$. Existen múltiples estudios y análisis que han investigado la problemática que existe en la implementación de los programas de detección del $\mathrm{CaCu}$ en México y en países de América Latina, cuyos resultados han documentado incumplimiento de normas de bioseguridad, deficiencia en la calidad de la toma de las muestras, errores en la interpretación de las pruebas y presencia de barreras socioculturales que dificultan que las mujeres acudan a dichos programas y permitan someterse a la toma de la citología cervical ${ }^{(7-10)}$.

Las mujeres de comunidades indígenas tienen los índices más altos de mortalidad por esta enfermedad, asociados a una detección tardía ${ }^{(11)}$. A pesar de ello, no existen en México cifras oficiales ni epidemiológicas relacionadas con el cáncer en esta 
población. Instituciones como el INEGI, la Comisión Nacional para el Desarrollo de los Pueblos Indígenas (CDI) y la Secretaría de Salud (SS) no cuentan con datos estadísticos ni epidemiológicos en torno al cáncer y su detección oportuna en población indígena ${ }^{(12)}$. No obstante, a pesar de que no existen fuentes fidedignas en torno a problemática, la mortalidad por $\mathrm{CaCu}$ en población indígena mexicana es mayor que el nivel nacional, el cual registra casi 14 muertes por cada 100 mil mujeres y en regiones indígenas casi se duplica ${ }^{(13)}$. El estado de Oaxaca, que concentra la mayor población indígena total en México con el 33,8\%, registró la tasa de mortalidad más alta en todo el país por $\mathrm{CaCu}$ con un 20.1/100.000 en el año $2010^{(14)}$, incluso mayor que la que se dio en África de 17,6/100.000 en el año $2008^{(1)}$. De acuerdo con un estudio realizado por García et al., ${ }^{(15)}$ los municipios en este estado en donde ocurrieron el mayor número de muertes por $\mathrm{CaCu}$ son Oaxaca de Juárez, San Juan Bautista Tuxtepec, Salina Cruz, Huajuapan de León y Juchitán de Zaragoza.

A pesar de la evidencia descrita, la investigación sobre las razones de una mayor mortalidad por $\mathrm{CaCu}$ en mujeres indígenas en México es escasa. Algunos estudios sugieren que dicha mortalidad se asocia con una detección tardía por falta de recursos económicos, pobreza y dificultad en el acceso a los servicios ${ }^{(16)}$. La literatura muestra que las barreras para la toma de la citología cervical, que pueden ser estructurales, psicosociales, culturales o de otra índole, varían en cada grupo social, debido a que cada grupo étnico tiene distintas y particulares características demográficas, sociales y culturales, que influyen en la asistencia a programas de detección oportuna de $\mathrm{CaCu}$, lo que impacta de manera importante en la morbilidad y mortalidad por esta enfermedad. En razón de la alta prevalencia de defunciones por $\mathrm{CaCu}$ en Juchitán, de las particularidades socioculturales que presenta la población zapoteca y de la necesidad de documentar algunos de los componentes psicosociales y estructurales que contribuyen u obstaculizan la ejecución de conductas preventivas de salud en este grupo étnico, se consideró estratégico estudiar las barreras que existen en esta región para la toma de la citología cervical.

Con base en lo anterior, en esta propuesta de investigación se propuso identificar las barreras de tipo estructural, psicosocial y cultural que influyen en la toma de la citología cervical en mujeres juchitecas, desde la voz del personal de salud encargado de estos programas de detección oportuna. La información obtenida permitió conocer algunas de las necesidades de atención en salud, enfocadas a la detección oportuna del $\mathrm{CaCu}$ en mujeres juchitecas y además aportó elementos importantes para próximos diseños de programas de intervención que ayuden a guiar la toma de decisiones en este campo y que contribuyan a la generación de políticas públicas a favor de la salud sexual de mujeres indígenas.

\section{Participantes y métodos}

El tipo de estudio fue exploratorio/descriptivo de corte cualitativo. La muestra quedó conformada por enfermeras y médicos de centros de salud públicos de primer nivel y médicos de Organizaciones No Gubernamentales (ONG's) que trabajan en programas de detección oportuna del $\mathrm{CaCU}$ en la comunidad. Se llevó a cabo un muestreo intencional ${ }^{(17)}$ se tomaron los casos que cumplieran con los criterios de inclusión (Profesionales de la salud encargados de la toma de 
la citología cervical en Juchitán), obteniendo un total de 8 participantes. De 10 clínicas del sector salud público existentes en la ciudad de Juchitán, se eligieron 5 por ser las que más afluencia de pacientes tenían. Se eligieron 2 clínicas privadas por accesibilidad y apertura al estudio. No hubo participantes que declinaran participar en este estudio.

En razón de que este estudio forma parte de un proyecto mayor, en esta fase la muestra quedó conformada por: (a) enfermeras del sector salud públicos $(\mathrm{n}=5)$ que trabajan en programas de detección oportuna del $\mathrm{CaCu}$, (b) encargados de los programas de detección oportuna $(\mathrm{n}=1)$, pasantes de enfermería (1) y (c) médicos encargados de los programas de detección oportuna en servicios privados $(\mathrm{n}=2)$ de organizaciones no gubernamentales (ONG's). En la tabla 1 se muestran las características de los participantes.

Tabla 1. Características principales de los participantes.

\begin{tabular}{|c|c|c|c|c|c|c|}
\hline Informante & Sexo & Edad & $\begin{array}{c}\text { Lugar de } \\
\text { Origen }\end{array}$ & $\begin{array}{c}\text { Estado } \\
\text { Civil }\end{array}$ & Puesto & $\begin{array}{l}\text { Tiempo en } \\
\text { programas } \\
\text { de tamizaje }\end{array}$ \\
\hline Enfermera 1 & $\mathrm{~F}$ & 29 & Juchitán & Casada & $\begin{array}{c}\text { Enfermera } \\
\text { Centro de } \\
\text { Salud }\end{array}$ & 9 años \\
\hline Enfermera 2 & $\mathrm{~F}$ & 25 & Juchitán & Casada & $\begin{array}{l}\text { Enfermera } \\
\text { Centro de } \\
\text { Salud }\end{array}$ & 2 años \\
\hline Pasante & $\mathrm{F}$ & 27 & $\begin{array}{l}\text { Santiago } \\
\text { Ixtaltepec }\end{array}$ & Casada & $\begin{array}{c}\text { Pasante } \\
\text { Servicio Social }\end{array}$ & 3 meses \\
\hline Enfermera 3 & $\mathrm{~F}$ & 48 & Juchitán & Separada & $\begin{array}{c}\text { Enfermera } \\
\text { Centro de } \\
\text { Salud }\end{array}$ & 7 años \\
\hline Enfermera 4 & $\mathrm{~F}$ & 54 & Juchitán & Casada & $\begin{array}{c}\text { Enfermera } \\
\text { Centro de } \\
\text { Salud } \\
\end{array}$ & 26 años \\
\hline Enfermera 5 & $\mathrm{~F}$ & 36 & Juchitán & Casada & $\begin{array}{c}\text { Enfermera } \\
\text { Centro de } \\
\text { Salud } \\
\end{array}$ & 10 años \\
\hline Médico SS & $\mathrm{M}$ & 57 & Tehuantepec & Casado & Médico SS & 30 años \\
\hline $\begin{array}{l}\text { Médico } \\
\text { Privado } 1\end{array}$ & M & 54 & Ixtaltepec & Soltero & Director ONG & 25 años \\
\hline $\begin{array}{l}\text { Médico } \\
\text { Privado } 2\end{array}$ & $\mathrm{~F}$ & 38 & Espinal & Soltera & $\begin{array}{c}\text { Coordinadora } \\
\text { del Programa } \\
\text { ONG }\end{array}$ & 10 años \\
\hline
\end{tabular}

El objetivo general fue identificar las barreras de tipo estructural, psicosocial y cultural que influyen en la toma de la citología cervical en mujeres juchitecas, percibidas por el personal de salud encargado de los programas de detección 
oportuna del $\mathrm{CaCu}$ en Juchitán. Se realizaron entrevistas semi-estructuradas a los participantes la cual indagó sobre: 1) Características Sociodemográficas: Se realizó un primer acercamiento para conocer y poder situar a los participantes en su contexto socioeconómico. 2) Experiencia de los Trabajadores de Salud en los programas de tamizaje del $\mathrm{CaCu}$ en mujeres juchitecas. Se realizaron preguntas que pudieran dar cuenta de su historia y experiencia como trabajadores de salud en programas de tamizaje en la comunidad, así como de las posibles dificultades percibidas para la realización de sus funciones, disponibilidad de recursos del sector salud y las barreras que según su opinión obstaculizan la asistencia de las mujeres juchitecas a la toma de la citología cervical. Las entrevistas tuvieron una duración de 45 a 60 minutos aproximadamente. Los contactos se hicieron en el mes de octubre del 2015, en tanto que las observaciones de campo y entrevistas tuvieron inicio en el mes de noviembre del mismo año. El procedimiento general que se utilizó para recabar la información, consistió en acudir a las clínicas y pedir hablar con el encargado para informarle de los objetivos del estudio; fue el mismo responsable de la clínica quien facilitó el contacto con las enfermeras y médicos encargados del programa. Se solicitó la colaboración voluntaria de los informantes para participar en el estudio mediante la firma de un consentimiento informado. Así mismo, se pidió a los participantes su autorización para grabar en video la entrevista, se les aclaró de manera previa que la información recabada era confidencial y con fines únicamente de investigación. Se tomaron las precauciones pertinentes para resguardar la intimidad de los participantes que integraron la muestra y la confidencialidad de su información personal.

Para el análisis de los datos, se transcribió a texto la información de las entrevistas. Tras la transcripción, se realizó el análisis de contenido en donde se elaboraron las categorías y la búsqueda de subcategorías para clasificar la información. Se usó el programa Atlas-ti. El contenido de las entrevistas se organizó en tres ejes temáticos: (a) barreras estructurales, (b) psicosociales y (c) culturales (tabla 2).

Tabla 2. Ejes Temáticos: Barreras estructurales, psicosociales y culturales para la toma de la citología cervical.

\section{Barreras Estructurales Barreras Psicosociales Barreras Culturales}

- Organización

- Presupuesto

- Información

- Conductas del Personal de Salud
- Información

- Características Sociodemográficas

- Actitudes/Emociones de la mujer
- Actitud de la pareja ante la prueba

- Tabú de la sexualidad

- Mitos

- Costumbres/cosmovisión

- Lengua

- Mitos/creencias

\section{Resultados}

Juchitán cuenta con un hospital de $2^{\circ}$ nivel, 8 centros de salud urbanos y 4 centros de salud rurales, de acuerdo con el documento oficial ${ }^{(18)}$. No obstante, según 
la Jurisdicción existen 10 clínicas en la ciudad de Juchitán. La mayoría de las localidades no cuenta con personal médico ni medicamentos para tratar las distintas enfermedades. Dentro del municipio de Juchitán se eligió a la ciudad de Juchitán para hacer el estudio, debido a que es cabecera municipal y muchos habitantes de las distintas localidades del municipio acuden a esta ciudad para recibir atención médica, ya que en sus localidades no cuentan con personal médico ni instalaciones adecuadas.

\subsection{Barreras}

Se consideró como barrera a toda circunstancia o condición que dificulte, obstaculice o impida la presencia de la conducta esperada: toma de la citología cervical. Las Barreras Estructurales tienen que ver con el sistema de salud y las instituciones. Las Barreras Psicosociales refieren procesos interpersonales y características sociodemográficas que dificultan o impiden la asistencia a la toma de la citología cervical. Las Barreras Culturales se vinculan con las normas que prevalecen en una comunidad que obstaculizan la asistencia a la toma de la citología cervical.

El contenido de las entrevistas se organizó, en categorías y subcategorías además de identificar la frecuencia con que fueron mencionadas cada una de las barreras identificadas.

Tabla 3. Barreras percibidas por el personal de salud

\begin{tabular}{|c|c|c|}
\hline \multicolumn{3}{|c|}{ Barreras Estructurales } \\
\hline Categorías & Subcategorías & $\mathbf{F}$ \\
\hline \multirow{3}{*}{ Organización } & $\begin{array}{l}\text { Fallas en los procesos en el envío de las muestras y la } \\
\text { obtención de resultados }\end{array}$ & 18 \\
\hline & No tomar la muestra a mujeres cuando llega al servicio & 14 \\
\hline & Procedimientos poco funcionales & 13 \\
\hline \multirow{3}{*}{ Presupuesto } & Falta de material & 16 \\
\hline & Falta de infraestructura & 8 \\
\hline & Falta de personal y de recursos humanos & 4 \\
\hline Información & $\begin{array}{l}\text { Desinformación del personal sobre procesos } \\
\text { administrativos }\end{array}$ & 5 \\
\hline \multirow{3}{*}{$\begin{array}{l}\text { Conductas del } \\
\text { Personal de Salud }\end{array}$} & $\begin{array}{l}\text { Falta de comunicación y enseñanza por parte del } \\
\text { personal médico a las usuarias. }\end{array}$ & 3 \\
\hline & Maltrato del personal & 2 \\
\hline & Descuidos en la toma de la muestra & 1 \\
\hline
\end{tabular}




\section{Barreras Estructurales}

\begin{tabular}{|c|c|c|}
\hline Categorías & Subcategorías & $\mathbf{F}$ \\
\hline \multicolumn{3}{|l|}{ Barreras Psicosociales } \\
\hline Información & $\begin{array}{l}\text { Desinformación por parte de la mujer acerca de la } \\
\text { prueba y de la enfermedad }\end{array}$ & 35 \\
\hline \multirow{4}{*}{$\begin{array}{l}\text { Características } \\
\text { Sociodemográficas }\end{array}$} & $\begin{array}{l}\text { Edad: Mujeres menores de } 25 \text { años con mayor pena a la } \\
\text { prueba, acuden con menor frecuencia }\end{array}$ & 4 \\
\hline & $\begin{array}{l}\text { Edad: Mujeres mayores de } 50 \text { años con mayor } \\
\text { resistencia a la prueba }\end{array}$ & 3 \\
\hline & Bajo nivel económico & 7 \\
\hline & Bajo nivel educativo & 2 \\
\hline \multirow{2}{*}{$\begin{array}{l}\text { Actitudes/Emociones } \\
\text { de la mujer }\end{array}$} & $\begin{array}{l}\text { Miedo (a que salga algo malo en la prueba, a que la } \\
\text { prueba sea dolorosa) }\end{array}$ & 7 \\
\hline & Falta de preocupación (desidia) & 3 \\
\hline \multicolumn{3}{|l|}{ Barreras Culturales } \\
\hline $\begin{array}{l}\text { Actitudes de la pareja } \\
\text { hacia la prueba }\end{array}$ & Prohibición, molestia de la pareja hacia la prueba & 17 \\
\hline Tabú de la Sexualidad & $\begin{array}{l}\text { Resistencia al análisis ginecológico, pena o vergüenza, } \\
\text { resistencia a pasar con médicos varones }\end{array}$ & 33 \\
\hline $\begin{array}{l}\text { Costumbres/ } \\
\text { Cosmovisión }\end{array}$ & Acuden a curanderas o parteras & 4 \\
\hline Mitos/Creencias & $\begin{array}{l}\text { Estigma y mitos del virus del papiloma humano, } \\
\text { creencia de que el cáncer es una "maldición" o } \\
\text { "brujería", creencia de que la prueba es dolorosa }\end{array}$ & 9 \\
\hline Lengua & Dificultades en la comunicación & 8 \\
\hline
\end{tabular}

\subsection{Barreras Estructurales}

Como lo muestra la tabla 3, se encontraron diversas barreras estructurales reportadas por el personal de salud, dentro de las más mencionadas se encuentran las que tienen que ver con la organización, como las fallas en los procesos de envío de las muestras y obtención de resultados; por ejemplo, la entrega de resultados es muy tardada, a veces tardan hasta tres meses en entregar el resultado de un examen citológico.

"[...] .... A veces las usuarias dicen "es que siento que tengo un problema, siento esto, siento otro, por favor si me lo puedes mandar urgente" sí, nosotros lo checamos que tiene alguna alteración, nosotros ponemos "revisado por patólogo y de manera 
urgente" Entonces a veces ya llega antes de los dos meses porque por lo regular tarda bastante. ¡A veces tres meses!...” (Enfermeras Centro de Salud). En otros casos se reportó que las muestras nunca llegan y que en ocasiones se traspapelan.

Otra de las barreras más comentadas por el personal de salud, corresponde al presupuesto, de lo que se deriva la falta de material, lo que fue reportado como uno de los principales obstáculos para realizar su trabajo [...] ...Pues damos el servicio a la gente, con lo que nosotros tengamos. Y algunas veces, no tenemos material, porque no hay... nosotros pedimos, hacemos requisiciones, y lo que hay en almacén es lo que nos dan, pero pues desgraciadamente, aún no hay todo... lo que más carecemos aquí son de guantes y pues tengo ahora si que ver que ya juntando ahi unos pesos pues se compra una caja y ya tenemos, con nuestros ingresos para poder comprarlo ¿no?..” (Enfermera Centro de Salud).

La falta de espacios específicos y designados para la toma de la citología fue una barrera reportada como importante, ya que les dificulta poder ejecutar sus funciones [...]...Para empezar no tenemos un área especifica, donde tomarlo, el centro de salud es pequeño y el área donde estaba blindada para toma de citologías, eh, está abandonada porque llegaron a reconstruir y lo abandonaron, o sea abandonaron la obra. Tuvimos que adaptar un espacio para una muestra de citología, de cierta manera eso complica un poco las cosas... uno como, como personal de enfermería, por lo menos a mi, eh, me gusta hacer un trabajo con calidad, es un área que implica calidad, un área adecuada, limpia, espaciosa. Y aquí, no la tenemos, entonces desde el momento que tú cierras la puerta con el usuario ya, ya estás dando una atención, y eso de que te toquen para "pásame el abate-lenguas" "¿está la toalla ahi??" Entonces, como vas a hacerle bien la entrevista al usuario si están tocando ¿no? Entonces, no es tan agradable trabajar así..." (Enfermera Centro de Salud).

\subsection{Barreras Psicosociales}

Con relación a las barreras psicosociales, lo más reportado fue la desinformación de las mujeres con respecto a la prueba, el VPH y los procesos de enfermedad. La desinformación relacionada a la prueba tiene que ver con el desconocimiento del procedimiento de la muestra, los beneficios y los requisitos que se necesitan para tomarla.

"[...] ....No tienen información de la muestra. En una ocasión tuve una paciente que dijo, - "también mi esposo viene a toma de Papanicolau” y yo dije... - "nada más es para las mujeres, porque es para detectar cáncer cervicouterino y no lo tienen, no tienen útero" Y ahí venia el pobre a hacerse su papanicolau también.....A veces son señoras de 30 - 40 años y es la primera vez que se lo van a hacer, entonces desconocen totalmente que se les va hacer..." (Enfermera de Centro de Salud).

De acuerdo a la información analizada, también se encontró que el personal de salud considera que las mujeres tienen desinformación respecto al proceso de enfermedad, no teniendo clara la evolución y el papel que juega el VPH, "[...] ... les falta información porque hemos visto casos de mujeres que llegan muy estresadas porque tienen el virus del papiloma y piensan que se van a morir..." (Enfermera de Centro de Salud).

También se mencionó la falta de información sobre la importancia de las prácticas preventivas, ya que las mujeres acuden al centro de salud hasta que presentan síntomas 
ginecológicos o cuando se sientes enfermas "[...] ... Yo encuentro a la señora que se niega rotundamente... ella dice - "aunque me lo diga mil veces, yo no voy a ir porque yo no siento nada, yo no tengo nada y yo no tengo porque ir a hacerme estudios..." (Médico Centro de Salud).

La cuestión económica también es vista como una barrera, sobre todo cuando necesitan agilizar la obtención de los resultados y las enfermeras envían a las mujeres a realizarlo a alguna clínica particular, ya que en el sector salud el tiempo de espera puede ser hasta de 3 meses, y muchas veces las mujeres no tienen los recursos para acudir a servicios privados. "[...]...Porque hay mujeres que están saliendo con Nic 1, con Nic 2 de 20 años...entonces de hecho se les toma la muestra pero de forma particular y pues estamos hablando de Juchitán, la mayoría no tiene la posibilidad de pagar una citología de forma particular..." (Enfermeras de Centro de Salud).

En el sector privado también se ve como un gran obstáculo la falta de recursos económicos para poder asistir por primera vez y de manera recurrente a la toma de la citología cervical "[... ... Bueno, siempre la dificultad que refieren es los costos y, sin embargo, los costos que se tienen son costos que consideramos que son accesibles en comparación con otros lugares privados ..." (Médico ONG).

Otro de los obstáculos mencionado por el personal de salud, fue el miedo que experimentan las mujeres, tanto el miedo a que les digan que tienen algo "malo", así como también el miedo a que les duela, "[...]...Como que sí se quieren hacer la prueba, pero como que a la vez no ¿no? Y eso como que es, una barrera entre nosotras como personal de salud y con las mujeres que son las usuarias ¿no?... muchas dicen, “'me va a doler?”...” (Enfermera de Centro de Salud).

Otra barrera percibida por el personal de salud fue la falta de preocupación de las mujeres hacia ellas mismas, aspectos más individuales que hacen que éstas no se interesen en acudir, la "desidia o dejadez" término que usaron reiteradas veces, "[...] ... yo lo veo por la desidia de ellas, - "Ay ya voy a hacerme mi papanicolau, ay ya, siempre no voy" ¿no? - "Y, y, luego tardan, y "luego no están” y "luego” ch, ah! "está lejos" "ay, luego voy y luego voy, los niños" luego entonces de cierta manera la gente también es muy desidiosa..." (Enfermera de Centro de Salud).

\subsection{Barreras Culturales}

Dentro de este rubro, se encontraron diversas barreras y obstáculos percibidos por el personal de salud, uno de los más mencionados tiene que ver con las actitudes de la pareja hacia la prueba, en donde se menciona, que ésta no permite a la mujer realizársela y que la mujer difícilmente acude o regresa por el miedo a que su pareja se entere, "[...] ... - "cómo voy a decirle a mi esposo, si él no sabe que vine a tomarme, es más él no quería, es más él me lo prohibió"... incluso hasta con los anticonceptivos, pues, vienen a escondidas. Pues sí, es una situación difícil..." (Enfermera de Centro de Salud).

En algunos casos la pareja está molesta y pide entrar a la prueba con la mujer; el personal de salud pública reportó que no los dejan entrar, “[...] ... Pues hay maridos que sí, son un poco celosos, o hay mucho machismo ¿no? que no las vean, o sea que sea una enfermera, menos un médico o menos un enfermero, que no sea hombre el que se lo tome, pero ahí vienen detrás de ellas..." (Enfermera de Centro de Salud).

El sector privado coincidió también en que la molestia y el enojo del esposo hacia la prueba es una barrera, a diferencia del sector público, ellos reportan dejar entrar a 
la pareja en aras de que la mujer pueda realizarse el estudio, " [...] ...pues eh, lo que a veces se presenta, es que las mujeres llegan acompañadas de su esposo, de su pareja y piden estar presentes en el estudio. Pero lo que hemos visto últimamente es que hay mujeres que le dicen a la pareja que se esperen en la sala. Y, sin embargo, hemos tenido casos que las mujeres no tiene otro recurso que aceptar que el marido o la pareja entre durante el estudio. Y lo que hacemos a veces es, en aras de que la mujer tenga acceso al servicio, aceptar que la pareja esté en el consultorio durante al estudio..." (Médico ONG).

El tabú de la sexualidad fue de los obstáculos más frecuentes reportado por el personal de salud, quienes lo distinguen como una barrera importante, ya que hay mucha resistencia al análisis ginecológico por sentimientos de pena, vergüenza o pudor. La censura y el control del cuerpo es una vivencia que se asocia a la dificultad para acudir a las pruebas de detección oportuna del $\mathrm{CaCu}$ " $[\ldots]$... le digo que son, se ponen muy nerviosas...hay mujeres que son muy nerviosas y penosas ¿no?... Y todavía "ay me da pena” asi exactamente con su pena (Enfermera de Centro de Salud). "[...]...Pues, la verdad, ellas manifiestan que es incómodo porque les da pena, les digo "mire, a mi también me da pena, yo soy mujer, porque a lo mejor no voy a, no me lo voy a tomar acá, pero yo voy a ir con mi ginecólogo o con mi ginecóloga, aunque sea mujer pues si da pena porque no la conoces ¿no?...” (Enfermera de Centro de Salud).

También reportaron que una gran barrera que tienen las mujeres es la resistencia a pasar con médicos u enfermeros varones y que piden pasar con mujeres, incluso llegan de otras clínicas buscando mujeres, el personal lo ve como un retraso y percibe que se debe enseñar a la mujer a que pase con médicos varones. " $[. .$.$] ...$ Les cuesta trabajo, seguimos así, a la gente todavía le cuesta trabajo darse cuenta o abrirse frente a un médico varón eh, desde, desde la sala de espera - ¿quién está dando consulta?” - "El Dr." - "híjole, ¿no me puedes pasar con la Dra.?... No, no podemos, no podemos pasarla con la Dra. pues, tenemos que nosotros, tenemos que enseñarla ¿no? Que no por el sexo es bueno o es malo el médico ¿no? Si no que el médico se va a enfocar a darte un resultado para tu mejoría. No nos importa, no le debe importar si es hombre o mujer la que la va a atender..." (Enfermera de Centro de Salud).

La lengua es otro gran obstáculo, ya que si el personal no habla zapoteco y las mujeres no hablan español, los problemas de comunicación dificultan la atención, la confianza y la asistencia. En los centros de salud no existen traductores oficiales, en algunas clínicas, el personal es de la región y habla o entiende el zapoteco, sin embargo, no pasa en todas. "[...] ...Sí, decírmelo a mí, que yo alguna vez me sentí muy mal cuando terminé de platicar con la señora - “¿me podría comentar lo que ya le platiqué?"... no pues no hablaba mi idioma, nada más me decía sí con la cabeza, es como si, si fuera un mudo pues. Y, y te ve y te dice que sí y a la hora que, no sabe expresar lo que tú hablas y si me sentí bien mal y desde ese tiempo ya veo a una persona y digo - "¿habla español?...” (Médico Centro de Salud).

Fueron notorios además, los prejuicios y mitos que imperan en relación a la enfermedad y el VPH, lo que dificulta la asistencia y el seguimiento, por ejemplo, el estigma que viven las mujeres solteras, las mujeres que tienen historia de varias parejas sexuales y las mujeres que tienen el VPH son consideradas como mujeres "sexoservidoras" "[...]...Por ejemplo en mis pláticas que he dado a muchas pacientes dicen que pensaban que ese virus no más lo tenían las señoras que se 
dedicaban a, a la prostitución, personas decían que nada más eran de ellas... Y, muchas personas, lo que he visto, es que no quieren que se entere otra persona que tiene el virus del papiloma les da pena..." (Médico ONG).

\section{Discusión}

La presente discusión se organiza en 3 secciones: 1) Acerca de las barreras estructurales, 2) Acerca de las barreras psicosociales y 3) Acerca de las barreras culturales.

\section{1) Barreras Estructurales}

Dentro de las barreras estructurales, en la categoría de organización se identificó la subcategoría fallas en los procesos en el envío de las muestras y la obtención de resultados y el código tardanza en la entrega de resultados fue común. Esto es consistente con otros estudios, el largo tiempo de espera para recibir el resultado del Pap., ha sido ampliamente documentado ${ }^{(19)}$ donde lo describen como uno de los principales obstáculos para que la mujer pueda continuar su atención. Los largos tiempos de espera en los centros y la tardanza en la entrega de resultados se relacionan con la cantidad insuficiente de especialistas, lo que puede traducirse a los pocos recursos destinados al diagnóstico y tratamiento de los resultados citológicos anormales en la comunidad indígena de Juchitán. Por lo que es fundamental, que se destinen recursos suficientes a estos programas. Se ha documentado que los tiempos de espera para ser atendidas y los pocos horarios establecidos para la toma de la muestra afectan de manera negativa la asistencia y seguimiento de las mujeres a los servicios de detección oportuna ${ }^{(20)}$. Esto es una situación que vulnera los derechos de las mujeres a la salud y a recibir los resultados en un tiempo prudente (4 semanas) ${ }^{(21)}$, lo que arrojó este estudio es que los tiempos de espera van hasta los tres meses para que se les entregue a las mujeres sus resultados. Las acciones estructurales y de los encargados para agilizar los tiempos son informales y no hay un procedimiento estable, además de tener pocos especialistas para la interpretación de las muestras en todo el Estado, enviando las tomas desde la región del Istmo hasta la ciudad de Oaxaca, puesto que no hay citotecnólogos en la región. El estructurar y analizar el procedimiento desde la toma de la muestra, la lectura y la entrega de resultados, así como las dificultades que existen en este proceso pueden implementar reformas para reducir los tiempos de espera.

Con relación a la subcategoría fallas en los procesos en el envío de las muestras y la obtención de resultados en la categoría de organización fueron la pérdida de seguimiento y el traspapeleo de las muestras. De acuerdo con Paolino et al., ${ }^{(19)}$ el hecho de que los servicios y los profesionales de salud no posean información relacionada con lo que pasa con el seguimiento y con el tratamiento de las mujeres con resultados anormales del Pap. que necesitan diagnósticos y tratamiento refleja importantes fallas en el monitoreo del proceso. El personal de salud informó que el seguimiento se pierde porque los resultados tardan y cuando llegan, van a visitar a las mujeres a sus casas para informarles de las anomalías; sin embargo, no siempre las encuentran, por lo que el seguimiento se pierde cuando es fundamental que se mantenga. Es importante que el personal de salud esté enterado y al pendiente del seguimiento de las pacientes e implementen otros procesos más funcionales. 
El personal de salud reportó como una barrera de organización, el hecho de no tomar la muestra a las mujeres cuando llegan al servicio, ya sea por la edad (menores de 25 y mayores 65 años de edad), por el horario, por pertenecer a otras clínicas, incluso en casos en que presentan factores y anomalías visibles, lo que dificulta que las mujeres regresen a los centros de salud, este resultado no ha sido reportado por otros investigadores, lo que refleja que en esta zona se vulneran los derechos de salud a las mujeres indígenas, ya que negar la atención es considerado un hecho discriminatorio. Algunos autores sugieren que brindar la facilidad para la toma de la prueba en las horas de atención ${ }^{(22)}$, acomodar las citas de acuerdo a los horarios en los cuales sea más fácil que las mujeres puedan acudir a realizarse el Pap ${ }^{(23)}$, mejorar los procesos y funciones administrativas de los centros de salud, como citar a las mujeres y notificarlas ${ }^{(24)}$ así como tomar la prueba a toda mujer que presente factores de riesgo y surtir el material necesario a las clínicas, puede ayudar a disminuir las barreras referentes a la toma de la prueba en mujeres juchitecas.

Otra de las barreras dentro de la organización informada por el personal de salud, fueron procedimientos poco funcionales del programa de detección oportuna, en especial el de captura de híbridos, en el cual el personal no tiene la información administrativa necesaria, además de que la requisición de material es complicada y poco práctica, ya que la jurisdicción no les brinda el material a las distintas clínicas sino que tienen una lista de (10-15 mujeres) que se vayan a realizar la prueba, el problema surge cuando acude la mujer a la cínica y le niegan la atención por no reunir el número mínimo de mujeres, por lo que las vuelven a citar y en muchos casos, las mujeres no regresan. Esto significa que las mujeres de 23 a 64 años de edad están descubiertas, puesto que en este rango de edad el SS tiene la instrucción de tomarles la captura de híbridos. Esto representa una gran falla estructural. Por otro lado se encontró que la prueba de captura de híbridos fue incorporada en los programas hace poco tiempo y cada clínica tiene información diferente sobre ésta. Este resultado es similar a lo reportado en Colombia ${ }^{(25)}$ quienes documentaron que se reemplazaron los programas ya estructurados a través de normas que dictaron acciones de carácter obligatorio. Sin embargo, se demostró que hay una amplia gama en la interpretación de las normas y reglas. Ello constituye una importante limitación por parte del personal del SS ya que el limitarse a lo que está estrictamente definido por la normariva favorece que el tratamiento que debe ser humano con respeto y calidad, se transforme en una actividad que raya en lo mecánico y despersonalizado.

Con relación a las fallas en los procesos, se encuentra la saturación de trabajo en las clínicas de salud pública, lo que fue reportado sólo por el personal de salud, sin embargo esto trae como consecuencia largos tiempos de espera o la necesidad de re-agendar a las mujeres, esto puede ocasionar que éstas no regresen. Estos obstáculos son resultado de la mala organización del sistema de salud y de la falta de recursos destinados a los programas de detección oportuna del $\mathrm{CaCu}^{(26)}$. Se ha documentado en otros estudios que el tiempo que espera una mujer para recibir una cita, el tiempo de espera para ser atendida y el hecho de que no haya suficientes horarios para la atención de acuerdo con la demanda se asocia de manera negativa a la oportunidad para un seguimiento(20).

Una barrera reportada por los participantes fue el presupuesto destinado para los programas de detección oportuna, lo que deriva en falta de infraestructura, 
personal especializado y tratamientos para el $\mathrm{CaCu}$ en la comunidad. De acuerdo al personal de salud y a las mujeres, existe falta de especialistas y personal preparado para la toma de la prueba en las clínicas del SS. El personal de salud además informó que padecen de falta de espacios específicos para la toma de las muestras y para dar pláticas educativas, así como falta de equipo para poder realizar su trabajo. Estos resultados son semejantes a lo reportado por Tejada-Tayabas et al., ${ }^{(27)}$ quienes en un estudio en San Luis Potosí, México, encontraron que los prestadores de servicios perciben como una debilidad del programa de detección oportuna del $\mathrm{CaCu}$ la falta de personal preparado en los centros de salud y la falta de un espacio específico para la toma de las muestras. Estas circunstancias incrementan la probabilidad de que se cite a las mujeres en fechas posteriores con el riesgo de que ya no regresen. Lo descrito evidencia dificultades para brindar una atención oportuna y provoca demoras en la atención a las mujeres, factor que facilita que la enfermedad avance. De acuerdo con Garcés et al., ${ }^{(28)}$ lo anterior se traduce a una violación a los derechos que tienen las mujeres a recibir un servicio digno y oportuno en el cuidado de su salud.

Dentro de las barreras estructurales informadas, se identificó la conducta del personal de salud como un obstáculo para la asistencia y la toma de la prueba, la relación entre los trabajadores de salud y las mujeres fue un factor comúnmente identificado como un obstáculo. En concreto, el mal trato del personal, un trato frío o poco empático y la falta de información, genera desconfianza y poca satisfacción por parte de las usuarias hacia los centros de salud y sus trabajadores. Esto ha sido reportado por Huamán ${ }^{(29)}$ quien describe que lo anterior puede influir en la decisión de las mujeres para no adoptar conductas preventivas para su salud y no acudir a los centros de detección oportuna del $\mathrm{CaCu}$. Por lo anterior, resulta fundamental y esencial que los trabajadores de la salud esten sensibilizados para brindar una atención de calidad, resolver las dudas de las mujeres juchitecas así como ser empáticos hacia sus temores, lo que en definitiva influirá en la satisfacción de las pacientes.

Lo descrito podría ser resultado de la burocracia tan compleja que está implícita en la organización del sistema de salud de Juchitán. Es fundamental mirar las desigualdades en salud en esta población, ya que el hecho de negarles la atención, no otorgar suficiente presupuesto para los programas y no ser sensibles a las particularidades de la población es un acto de abandono y discriminatorio.

\section{2) Barreras Psicosociales}

Con relación a las barreras psicosociales, la edad, fue reportada por el personal de salud, sin embargo, no llegaron a un acuerdo relacionado a si las mujeres de generaciones más jóvenes o más mayores son las que tienen mayor resistencia a la prueba. Por lo que, en la presente investigación, se evidencia inconsistencia en considerar a la edad como barrera para la toma del Pap. En contraste, la edad ha sido ampliamente documentada como un factor asociado a la toma del Pap, en Estados Unidos por ejemplo, el Instituto Nacional del Cáncer ${ }^{(30)}$ informó que más de la mitad de las mujeres mayores de 65 años no se habían realizado la citología cervical en los últimos 3 años. Al respecto Medrano ${ }^{(31)}$ encontró que la edad de las mujeres de entre 20 y 29 años se asocia con la negación a la toma del Papanicolaou. Por lo anterior, se deduce que no hay un convenio referente a la edad y la toma del 
Pap, esto puede explicarse por las diferencias culturales y sociales en cada grupo de mujeres investigadas, por lo que se requieren programas de detección oportuna enfocados a cada grupo de edad.

Otra de las barreras relacionada con las características sociodemográficas fue el bajo nivel educativo, misma que se relaciona con una pobre asistencia a la toma del Pap. Esto es importante debido a que en la ciudad de Juchitán existen 10.063 indígenas mayores de 15 años ${ }^{(32)}$ con analfabetismo. Este resultado es consistente con lo que informan otros estudios ${ }^{(33)}$ en donde encontraron que el bajo nivel educativo está asociado con pobre conocimiento y con una actitud negativa hacia la toma de la citología cervical.

El bajo nivel económico dentro de las características sociodemográficas, fue visto como una importante barrera, los participantes reportaron que las mujeres no tienen los recursos para pagar un médico particular y no tienen otra opción más que utilizar los servicios públicos, sin embargo, en muchas ocasiones las clínicas no tienen el material necesario, o no les toman la prueba por la edad, por lo que las envían a servicios particulares con el riesgo de que las mujeres no puedan pagar esos servicios y no se realicen la prueba. Los trabajadores de la salud identificaron además que las limitaciones económicas influyen para que las mujeres no regresen a los servicios de salud y a su seguimiento. En otros estudios se ha explorado si la influencia del ingreso económico tiene algún impacto en la decisión de realizar el Pap. La evidencia muestra resultados opuestos, en algunos estudios se ha observado que no hay influencia ${ }^{(31,34)}$ a diferencia de otros resultados en los cuales si han encontrado asociación significativa entre el índice de riqueza (de medio a muy rico) con la aceptación de la prueba ${ }^{(35,36)}$. Este factor es muy importante ya que la presente investigación documentó que en la población zapoteca de Juchitán existen características sociales específicamente económicas que dificultan e impiden la toma del Pap. Algunos autores ${ }^{(37)}$ sugieren que los medios de información masiva y algunas otras estrategias educativas son importantes, pues se ha visto que tienen la capacidad de aumentar la tasa de respuesta para acceder a los servicios médicos preventivos, sin importar la desventaja económica de la población.

Se ha de tomar en cuenta que una de las categorías que emergió más frecuentemente del análisis de contenido fue la percepción de los trabajadores de salud acerca de la falta de conocimiento que las mujeres juchitecas tienen sobre la enfermedad, el VPH, la prueba, el procedimiento y los beneficios. Estos resultados concuerdan con diversos estudios ${ }^{(38-40)}$ en los cuales han detectado que la falta del conocimiento relacionado con la prueba, es uno de los principales obstáculos por los que las mujeres no se la toman. Además se ha evidenciado que hay malos entendidos sobre el significado de la prueba y la enfermedad, lo que dificulta además la comunicación entre las mujeres y los profesionales de la salud ${ }^{(41)}$. Lo anterior nos lleva a pensar que la falta de información es un problema real, que necesita ser abordado para aumentar el nivel de conocimiento acerca de la importancia de la prueba, haciendo un mayor énfasis en las mujeres que no se lo han realizado. Sin embargo, es importante mencionar que los trabajadores de la salud, de alguna manera culpan a las mujeres por no estar informadas, cuando la falta de conocimiento puede deberse en gran medida a la falta de eficacia de los programas de difusión por parte del Estado y del sector salud, por lo que es fundamental poner atención y esfuerzo en la manera en que se están realizando dichos programas. Con relación a la falta de información y a las redes de comunicación, se ha reportado que proveer información, incrementar el conocimiento 
y la conciencia acerca del $\mathrm{CaCu}$ y estimular conductas saludables y preventivas en las mujeres en riesgo con edades de 30 a 50 años de edad, en particular en lugares donde los recursos de salud son limitados, resulta eficaz ${ }^{(42)}$.

Fue común la percepción del desconocimiento de síntomas como una barrera. Los participantes reportaron que para las mujeres, el $\mathrm{CaCu}$ es una enfermedad asintomática y no acuden a los servicios médicos si no presentan algún síntoma como dolor, sangrado o flujo vaginal. Sin embargo, la ausencia de síntomas asociados a los primeros estadios de la enfermedad es importante, ya que ha sido documentado que las mujeres no asisten o tienden a aplazar la toma del Pap cuando se sienten bien, lo que ha sido descrito como una manera de procrastinación ${ }^{(43-45)}$. El hecho de que las mujeres acudan a los servicios sanitarios sólo cuando aparece un problema de salud o se sienten enfermas se ha considerado como un acto negligente y de descuido ${ }^{(44,46)}$. Sin embargo, no hay que olvidar que también hay fallos estructurales, los cuales tienen que ver con los programas de difusión y con la información que el personal de salud debe dar a las mujeres, ya que el hecho de considerarlo un acto de descuido sólo por parte de las mujeres, implica no responsabilizar a otros actores sociales como los servidores de salud que forman también, una parte importante del problema. En este contexto, la calidad y pertinencia de la educación que se promueva tanto por parte del personal de salud como por las instituciones gubernamentales sobre los beneficios y propósitos de la prueba, así como de las características de la enfermedad, pueden ser estrategias pertinentes para derribar estas barreras.

Otra barrera identificada fue el miedo a tener algo "malo" como un cáncer, lo que coincide con otros estudios en los que se reporta que las mujeres no acuden por experimentar miedo ${ }^{(47)}$. Por ejemplo, el miedo a que les den un diagnóstico de cáncer también ha sido descrito por otros investigadores ${ }^{(48,49)}$. Es importante mencionar que la información relacionada con los posibles resultados que pueda arrojar la prueba, pueden disminuir el miedo de la mujer, ya que no siempre son negativos y si hay resultados de alguna patología pre-cancerosa, existen tratamientos para evitar el desarrollo de dicha enfermedad. Por lo que diseñar campañas informativas adecuadas es primordial. Por otro lado, el miedo al dolor también ha sido investigado ${ }^{(50)}$. En esta investigación fue común que el personal de salud, reportara que no acuden por miedo al dolor que les pueda generar la prueba, lo que obstaculiza el seguimiento y futuras tomas. Llama la atención la coincidencia de los profesionales de la salud con lo informado en estudios previos respecto a que el miedo al dolor proviene de la falta de conocimiento asociado al procedimiento ${ }^{(40)}$; sin embargo, es de suma importancia tomar en cuenta la experiencia de las mujeres ante la prueba, por lo que es fundamental que los centros de salud y los trabajadores que atienden a las mujeres juchitecas, sean sensibles ante el tema para prevenir situaciones de incomodidad y brindar mayor confianza a las usuarias.

\section{3) Barreras Culturales}

Con relación a las barreras culturales, una de las categorías que fue percibida por los informantes como una limitante para que las mujeres acudan a la toma del Pap, fue la actitud negativa de las parejas ya que no permiten que las mujeres acudan a estos programas. Estos resultados fueron consistentes con los arrojados por Huamán ${ }^{(51)}$ y Tamayo et al., ${ }^{(52)}$ quienes encontraron que un factor cultural para 
que las mujeres no accedieran a la toma de la prueba fue la oposición por parte del marido, lo cual tiene una gran influencia en la mujer, puesto que la reacción de la pareja puede ejercer cierto grado de violencia en cualquiera de sus tipos. Este rechazo fue detectado en esta investigación, especialmente en parejas de mayor edad. De esta manera se considera prudente incluir a los esposos en las campañas de difusión y de información, para lograr una actitud más favorable ante la prueba, además se ha visto que si ellos saben que su salud está en juego, manifiestan mayor importancia a la prueba ${ }^{(53)}$.

En cuanto al tabú de la sexualidad los trabajadores de salud manifestaron que los sentimientos de vergüenza, pena y pudor son comunes como limitantes para la prueba. La vergüenza que se presenta se asocia a la exposición de los genitales durante la realización del Pap (los médicos de este estudio reportaron que las mujeres presentan dificultad para bajarse la ropa interior $)^{(54-56)}$. Estas situaciones de vergüenza y pena podrían suavizarse si los profesionales de la salud les brindaran una atención empática y cuidadosa. El poco cuidado por parte de éstos hacia las mujeres, ha sido reportado como una barrera por otros autores ${ }^{(57)}$. De igual manera, en esta investigación identificaron como un obstáculo, ser atendidas por profesionales varones, lo que aumenta la vergüenza que las mujeres sienten ante el procedimiento, hecho que también ha sido reportado en otros estudios como una barrera ${ }^{(40,58)}$. Es importante tener en cuenta que si las mujeres no se sienten cómodas con el personal de salud o con el entorno, es difícil que ellas quieran regresar a conocer los resultados o accedan a ajustarse a algún seguimiento. Por ello, se sugiere una mayor educación sexual en casa y escuelas, así como fomentar la confianza que generen los profesionales de salud al promover que sean mujeres quienes tomen las pruebas ${ }^{(59)}$.

Los participantes reportaron el uso común de medicina tradicional y de parteras. Sin embargo, es importante aclarar, que los servidores de salud percibieron dichas prácticas tradicionales como una barrera para que las mujeres acudan a la toma del examen, puesto que la primera opción de apoyo que buscan es la medicina tradicional, lo que hace probable el atraso en el diagnóstico oportuno. De igual manera, se evidenció que las parteras intervienen no sólo en el embarazo y parto, sino también en otro tipo de enfermedades, lo que fue reportado también en otro estudio $^{(60)}$ donde se documentó el reconocimiento de su autoridad en la comunidad. Es comprensible entonces que la opinión de las parteras sobre las enfermedades y su tratamiento, juega un papel importante en la comunidad. En el estudio de Chavez y $\operatorname{Voss}^{(60)}$ las parteras de la cultura maya en Quintana Roo, envían a sus pacientes a los centros médicos, sin embargo, los profesionales de la salud no lo hacen y no aceptan un tratamiento complementario. En la comunidad juchiteca no se ha investigado la comunicación entre parteras y trabajadores de la salud, lo que es importante, tras la evidencia de que éstas tienen un reconocimiento social.

Fueron comunes los prejuicios y estigmas que imperan en la sociedad juchiteca, los cuales se reportaron por el personal de salud, como el prejuicio hacia las mujeres solteras que acuden a estos programas, lo que limita a éstas a asistir. Byrd et al., ${ }^{(61)}$ encontraron que uno de los obstáculos para que se realizaran el Pap fue la idea de que las mujeres solteras que se hicieran la prueba serían vistas como mujeres sexualmente activas. Lo anterior en una comunidad como Juchitán en donde aún existen prácticas que enaltecen la virginidad femenina como un requisito para el matrimonio, se vuelve una barrera fundamental. 
Las barreras idiomáticas fueron reportadas como un obstáculo, lo que es consistente con otros estudios, los cuales informan que las mujeres pertenecientes a alguna etnia indígena en Perú comparten dichas barreras ${ }^{(62,63)}$ éstas disminuyen la capacidad de la comunicación efectiva sobre la importancia de la toma de la prueba para la detección oportuna del $\mathrm{CaCu}$. En este sentido, se ha reportado que para lograr un correcto intercambio de información y conocimiento, es fundamental que los medios masivos de comunicación ${ }^{(62)}$ e incluso los programas de salud, sean congruentes y sensibles al nivel sociocultural de la comunidad indígena a la que se dirigen. Además, resulta evidente que si bien los profesionales de la salud no hablan el zapoteco, existan traductores dentro del sector salud, así como que sean sensibilizados acerca de las particularidades de la población para poder entablar un ambiente de confianza.

\section{Conclusiones}

El análisis temático identificó que los factores estructurales, psicosociales y culturales se relacionan entre sí y es difícil separarlos, estos interactúan constantemente influyendo en la asistencia a la toma de la citología cervical. Se identificaron barreras de tipo estructural, psicosocial y cultural para la toma de la citología cervical a partir del discurso de los participantes. Los principales obstáculos identificados por el personal de salud a nivel estructural fueron, la falta de material, de espacios apropiados para la toma y recursos humanos, la tardanza en la entrega de resultados y la desinformación dentro del sistema de salud y desde este nivel hacia la comunidad. Por otro lado, existen varios aspectos psicosociales y culturales que dificultan la asistencia de primera vez y regular a los servicios de detección oportuna del $\mathrm{CaCu}$, principalmente la desinformación, el miedo, el tabú de la sexualidad y la actitud negativa de la pareja hacia la prueba. Además, fueron comunes los prejuicios y mitos que rodean la enfermedad y el VPH, lo que cohíbe que la mujer se interese y acuda.

Se enfatiza a través del discurso de los participantes que existe desinformación relacionada al $\mathrm{CaCu}$ y a los programas de detección oportuna, a pesar de que muchas mujeres "han escuchado" hablar de esta enfermedad, gran porcentaje de ellas desconoce factores de riesgo, signos y síntomas del padecimiento y métodos de detección. Lo anterior indica que el mensaje no está llegando del todo claro a esta población y que existen algunas dificultades en la transmisión y recepción de la información, por lo que es indispensable investigar este punto. Por ello, se sugiere la inclusión de traductores en el sistema de salud, campañas informativas bilingües, difusión del programa en dependencias médicas rurales, así como derribar las barreras estructurales que se refieren al sistema de salud. De igual manera es fundamental dar voz a las mujeres para identificar cuál es su percepción en relación al tema.

A través de este estudio se observó que en Juchitán las mujeres se enfrentan a distintas y múltiples barreras para acudir a la toma de la citología cervical, sin embargo, estos obstáculos podrían ser tratados a través de información adecuada y educación para la salud. Es importante tomar en cuenta las condiciones específicas de esta población, ya que la información y educación para la salud tendría que ser adaptada a su lengua, cosmovisión y sus necesidades particulares. 


\section{Referencias bibliográficas}

1. GLOBOCAN. Estimated Cancer Incidence, Mortality and Prevalence Worldwide in 2012. [Acceso 15 de junio de 2017]. Disponible en: http://globocan.iarc.fr/Pages/fact sheets_cancer.aspx

2. IARC, Word Cancer Report 2008. [Acceso 15 de junio de 2017]. Disponible en: http:// www.iarc.fr/en/publications/pdfs-online/wcr/2008/

3. Instituto Nacional de Estadística y Geografía. Causas de defunción. Defunciones generales de mujeres por principales causas de mortalidad, 2012. [Acceso 15 de junio de 2017]. Disponible en: http://www3.inegi.org.mx/sistemas/sisept/Default. aspx $? \mathrm{t}=\mathrm{mdemo} 125 \& \mathrm{~s}=\mathrm{est} \& \mathrm{c}=23589$

4. The World Health Report. Bridging the gaps. World Health Organization, Geneva. 1995. [Acceso 17 de junio de 2017]. Disponible en: http://www.who.int/whr/1995/en/whr95_en.pdf

5. Centro Nacional de Equidad de Género y Salud Reproductiva. Manual de Procedimientos para la Toma de la Citología Cervical. 2006. [Acceso 17 de junio de 2017]. Disponible en: http://cnegsr.salud.gob.mx/contenidos/descargas/CaCu/toma_muestra.pdf

6. Lazcano EC, Moss S, Alonso de Ruiz P, Salmerón J, Hernández M. Cervical cancer screening in developing countries: why is it inefecctive? The case of Mexico. Arch Med Res 1999; 30:240-50. doi:10.1016/S0188-0128(99)00006-8

7. Salinas ANA, Villarreal E, Garza ME, Fraire J, López JJ, Barboza O. Calidad del programa de detección oportuna de cáncer cervicouterino en el estado de Nuevo León. Salud pública de México 1997; 187:194-39.

8. Alonso P, Lazcano EC, Hernández M. Cáncer cervicouterino, diagnóstico, prevención y control. México: Panamericana 2000; p. 155:174.

9. Hidalgo AC. El cáncer cérvico-uterino, su impacto en México y el porqué no funciona el programa nacional de detección oportuna. Rev Biomed 2006; 17: 81-4.

10. Noreña C, Tamayo LS. Cáncer de cuello uterino: análisis de la calidad de un programa. Aquichan 2010; 10: 52-68.

11. Vasilevska M, Ross SA, Gesink D, Fisman, DN. Relative risk of cervical cancer in indigenous women in Australia, Canada, New Zealand, and the United States: a systematic review and meta-analysis. J Public Health Policy 2012; 33:148-64. doi: 10.1057/jphp.2012.8.

12. Saldaña M, Ascencio L, Rangel N. Características de la población indígena en méxico en cuidados paliativos. Medicina, Salud y Sociedad 2014; 4: 268:84.

13. "Estrategia de Prevención Integral del Cáncer Cérvico Uterino" 30 municipios del Estado de Oaxaca con el mayor riesgo epidemiológico 2011. [Acceso 15 de junio de 2017]. Disponible en: http://www.programassociales.org.mx/sustentos/Oaxaca518/archivos/ lineamientos_VPH_vacunacion_municipios.pdf

14. Organización Panamericana de la Salud. Indicadores Básicos 2010. [Acceso 15 de junio de 2017]. Disponible en: http://www1.paho.org/mex/dmdocuments/indicadoresMEX2010. pdf

15. García MI, Mayoral M, Pintor JR, Malvaez N., Méndez PM, Sánchez M, et al. Perfil epidemiológico de la mortalidad por cáncer cervicouterino en Oaxaca 2000-2010 Rev Eviden Invest Clin 2011; 4: 10-14. [Acceso 15 de junio de 2017]. Disponible en: http:// www.medigraphic.com/pdfs/ evidencia/ eo-2011/eo111e.pdf

16. Condon JR, Barnes T, Cunningham J, Armstrong BK. Long-term trends in cancer mortality for Indigenous Australians in the Northern Territory. Med J Aust 2004; 180: 504-7. 
17. Kerlinger F, Lee H. Investigación del Comportamiento. Métodos de Investigación en Ciencias Sociales. $4^{\mathrm{a}}$ ed. México: McGraw-Hill, 2002.

18. Plan de Desarrollo Municipal de Juchitán de Zaragoza 2014-2016. [Acceso 15 de junio de 2017]. Disponible en: http://www.juchitandezaragoza.gob.mx/transparencia/archivos/ Plan\%20de\%20desarrollo\%20municipal\%20de\%20juchitan\%20de\%20zaragoza.pdf

19. Paolino, M, Pantelides E, Bruno M, Maceira V, Peña L, Godoy J, Arrossi S. Determinantes sociales del seguimiento y tratamiento de mujeres con Pap anormal en Avellaneda, Provincia de Buenos Aires. Rev Argentina Salud Pública 2011;2:21-7.

20. Aguero L. Análisis de la efectividad, oportunidad, accesibilidad y satisfacción de la usuaria en el programa de detección temprana del Cáncer de Cérvix según el Sistema Público de Salud y los servicios brindados por terceros en el I Nivel de Atención. Tesis Doctoral. Enero a Junio 2006/. [Acceso 15 de junio de 2017]. Disponible en: http:// biblioteca.icap.ac.cr/BLIVI/TESIS/2007/Aguero_Sandi_Lorena_2007_SA.pdf

21. Organización Mundial de la Salud. Comprehensive Cancer Control. A guide to essential practice. OMS Ginebra 2006. [Acceso 15 de junio de 2017]. Disponible en: http://apps. who.int/iris/bitstream/10665/144785/1/9789241548953_eng.pdf

22. Johnson, CE, Mues KE, Mayne SL, Kiblawi AN. Cervical cancer screening among immigrants and ethnic minorities: a systematic review using the Health Belief Model. J Low Genit Tract Dis 2008; 12:232-41. doi: 10.1097/LGT.0b013e31815d8d88

23. Valenzuela MT, Miranda A.¿Por qué NO me hago el Papanicolaou? Barreras sicológicas de mujeres de sectores populares de Santiago de Chile. Rev Chil Salud Pública 2001;5:75-80.

24. Abercrombie PD. Improving adherence to abnormal Pap smear follow-up. J Obstet Gynecol Neonatal Nurs 2001;30:80-8. doi.10.1111/j.1552-6909.2001.tb01524.x

25. Wiesner-Ceballos C, Murillo Moreno RH, Piñeros Petersen M, Tovar-Murillo SL, Cendales Duarte R, Gutiérrez MC. Control del cáncer cervicouterino en Colombia: la perspectiva de los actores del sistema de salud. Rev Panam Salud Publica. 2009;25:1-8. [Acceso 15 de junio de 2017]. Disponible en: http://www.scielosp.org/scielo.php?script=sci_arttext\& pid=S1020-49892009000100001

26. Murillo R. Control del cáncer de cuello uterino en Colombia: triunfos y desafíos de la tamización basada en la citología cérvico-uterina. Biomédica 2008;28:467-70. [Acceso 15 de junio de 2017]. Disponible en: https://www.revistabiomedica.org/index.php/ biomedica/article/view/52/379

27. Tejada LM, Hernández LE, Pastor MP. Fortalezas y debilidades del programa para la detección y el control del cáncer cervicouterino. Evaluación cualitativa en San Luis Potosí, México. Gac Sanit 2012;26:311-6.

28. Garcés IC, Rubio DC, Ramos SM. Barreras y facilitadores del sistema de salud relacionadas con el seguimiento de anormalidades citológicas, Medellín-Colombia. Gerencia y Políticas de Salud 2014;13:200-11.

29. Huamán M. Determinantes administrativos, psicológicos y culturales en la actitud hacia la prueba citológica de cuello uterino de mujeres trujillanas. UCV -Scientia 2010:2:3442. [Acceso 15 de junio de 2017]. Disponible en: file://C:/Users/RED/Downloads/4331359-1-PB\%20(1).pdf

30. Instituto Nacional de Cáncer de los Estados Unidos (s/f). [Acceso 15 de junio de 2017]. Disponible en: http//www.nci.nih.gov

31. Medrano MM. Factores socioculturales y psicológicos que influyen en la decisión de las usuarias para la toma de papanicolaou. Centro de Salud Carlos Cueto Fernandini-2014. 2015. [Acceso 15 de junio de 2017]. Disponible en: http://cybertesis.unmsm.edu.pe/ handle/cybertesis/4055 
32. Comisión Nacional para el Desarrollo de los Pueblos Indígenas. Indicadores sociodemográficos de la población total y la población indígena por municipio. 2000. [Acceso 15 de junio de 2017]. Disponible en: http://www.cdi.gob.mx/cedulas/2000/ OAXA/20043-00.pdf

33. Huamaní C, Hurtado A, Guardia M, Roca J. Conocimientos y actitudes sobre la toma de papanicolau en mujeres de Lima, Perú 2007. Rev Peru Med Exp Salud Pública 2008;25:44-50. [Acceso 15 de junio de 2017]. Disponible en: http://www.scielo.org.pe/ pdf/rins/v25n1/a07v25n1.pdf

34. Farfán LC, Barrón E, Jiménez ME, Vázquez E, Ayala SS. Factores socioculturales en mujeres que no acuden a detección oportuna de cáncer cervicouterino. Revista de Enfermería del Instituto Mexicano del Seguro Social 2004;12:123-30.

35. Gutiérrez C, Romaní F, Ramos J, Alarcón E, Wong P. Factores asociados con el conocimiento y tamizaje para cáncer de cuello uterino (examen de Papanicolaou) en mujeres peruanas en edad fértil. Análisis del periodo 1996-2008. Rev Peru Epidemiol [Internet]. 2010 [Acceso el 20 diciembre del 2012];14(1):[11 p.]. Disponible en: http:// redalyc.uaemex.mx/src/inicio/ ArtPdfRed.jsp?iCve=203119805006\#Quintana MRF. Factores que promueven la práctica de la detección oportuna de cáncer cervicouterino en mujeres adultas (Master dissertation, Universidad Autónoma de Nuevo León). 2000.

36. Orians CE, Erb J, Kenyon KL, Lantz PM, Liebow EB, Joe JR, Burhansstipanov L. Public education strategies for delivering breast and cervical cancer screening in American Indian and Alaska Native populations. J Public Health Manag Pract 2004;10:46-53.

37. Ogedegbe, G., Cassells, A. N., Robinson, C. M., DuHamel, K., Tobin, J. N., Sox, C. H., \& Dietrich, A. J. Perceptions of barriers and facilitators of cancer early detection among low-income minority women in community health centers. J Natl Med Assoc 2005;97:162-70.

38. Markovic M, Kesic V, Topic L, Matejic B. Barriers to cervical cancer screening: a qualitative study with women in Serbia. Soc Sci Med 2005;61:2528-35. doi: 10.1016/j. socscimed.2005.05.001

39. Urrutia MT, Poupin L, Concha X, Viñales D, Iglesias C, Reyes V. ¿Por qué las mujeres no se toman el Papanicolaou?: Barreras percibidas por un grupo de mujeres ingresadas al programa de cáncer cervicouterino AUGE. Rev Chil Obstet Ginecol 2008;73:98-103. Doi:10.4067/S0717-75262008000200005 [Acceso 15 de junio de 2017]. Disponible en: http://www.scielo.cl/pdf/rchog/v75n5/art02.pdf

40. Blake DR, Weber BM, Fletcher KE. Adolescent and young adult women's misunderstanding of the term Pap smear. Arch Pediatr Adolesc Med 2004;158:966-70. doi:10.1001/archpedi.158.10.966.

41. Herdman C, Sherris J. Planning appropriate cervical cancer prevention programs. 2000.

42. Wiesner C, Cendales R, Murillo R, Piñeros M, Tovar S. Seguimiento de mujeres con anormalidad citológica de cuello uterino, en Colombia. Rev Salud Pública 2010;12:1-13. [Acceso 15 de junio de 2017]. Disponible en: http://www.scielo.org.co/pdf/rsap/v12n1/ v12n1a01.pdf

43. Agurto I, Bishop A, Sanchez G, Betancourt Z, Robles S. Perceived barriers and benefits to cervical cancer screening in Latin America. Prev Med 2004;39: 91-8. doi:10.1016/j. ypmed.2004.03.040

44. Van Til L, MacQuarrie C, Herbert R. Understanding the barriers to cervical cancer screening among older women. Qual Health Res 2003;13:1116-31. doi:10.1177/1049732303255975

45. Goldman RE, Risica PM. Perceptions of breast and cervical cancer risk and screening among Dominicans and Puerto Ricans in Rhode Island. Ethn Dis 2004;14:32-42. 
46. Abercrombie PD. Improving adherence to abnormal Pap smear follow-up. J Obstet Gynecol Neonatal Nurs 2001;30:80-8. doi:10.1111/j.1552-6909.2001.tb01524.x

47. Byrd TL, Chavez R, Wilson, KM. Barriers and facilitators of cervical cancer screening among Hispanic women. Ethn Dis 2007;17:129-34. [Acceso 15 de junio de 2017]. Disponible en: https://www.columbus.gov/uploadedFiles/Public_Health/Content_Editors/ Community_Health/Minority_Health/Hispanic\%20women.pdf

48. Smith M, French L, Barry HC. Periodic abstinence from Pap (PAP) smear study: women's perceptions of Pap smear screening. Ann Fam Med 2003;21:203-08. [Acceso 15 de junio de 2017]. Disponible en: http://www.annfammed.org/content/1/4/203.full.pdf + html

49. Ybarra JL, Pérez BE, Romero D. Conocimiento y creencias sobre la prueba de Papanicolaou en estudiantes universitarios. Psicología y Salud 2013;22(2):185-194. [Acceso 15 de junio de 2017]. Disponible en: http://revistas.uv.mx/index.php/psicysalud/article/view/532/903

50. Huamán M. Determinantes administrativos, psicológicos y culturales en la actitud hacia la prueba citológica de cuello uterino de mujeres trujillanas. UCV -Scientia 2010;2:3442. [Acceso 15 de junio de 2017]. Disponible en: file://C:/Users/RED/Downloads/433$1359-1-\mathrm{PB} \% 20(2) \cdot \mathrm{pdf}$

51. Tamayo LS, Chávez MG, Henao LM. Cáncer de cuello uterino: más allá de lo que es; la percepción de las mujeres de Antioquia (Colombia) y Colima (México), 2008. Rev Fac Nac Salud Pública 2009;27:177-86. Disponible en: http://www.scielo.org.co/pdf/rfnsp/ v27n2/v27n2a09.pdf

52. Zelaya FB, Munguía ML. Barreras para la realización de la citología cervical en mujeres del Municipio de Telpaneca. SILAIS Madriz, julio noviembre del 2007 (Doctoral dissertation). 2008. [Acceso 15 de junio de 2017]. Disponible: http://riul.unanleon.edu. ni:8080/jspui/bitstream/123456789/2631/1/213927.pdf

53. Ho V, Yamal JM, Atkinson EN, Basen-Engquist K, Tortolero-Luna G, Follen M. Predictors of breast and cervical screening in Vietnamese women in Harris County, Houston, Texas. Cancer nursing 2005;28:119-29.

54. Fernández ME, Cardenas M. Cervical cancer screening among Latinas recently immigrated to the United States. Prev Med 2004;38:529-35. doi:10.1016/j.ypmed.2003.12.009

55. Smith M, French L, Barry HC. Periodic abstinence from Pap (PAP) smear study: women's perceptions of Pap smear screening. Ann Fam Med 2003;1: 203-208. [Acceso 15 de junio de 2017]. Disponible en: http://www.annfammed.org/content/1/4/203.full.pdf +html

56. Wiesner C, Vejarano M, Caicedo, JC, Tovar SL, Cendales R. La citología de cuello uterino en Soacha, Colombia: representaciones sociales, barreras y motivaciones. Rev Salud Pública 2006;8:185-96. [Acceso 15 de junio de 2017]. Disponible en: http://www. scielosp.org/pdf/rsap/v8n3/v8n3a04

57. McFarland DM. Cervical cancer and Pap smear screening in Botswana: knowledge and perceptions. Inter Nurs Rev 2003;50:167-75. Doi: 10.1046/j.1466-7657.2003.00195.x

58. Lazcano EC, Castro R, Allen B, Nájera P, de Ruiz PA, Hernandez M. Barriers to early detection of cervical-uterine cancer in Mexico. J Women's Health 1999;8:399-408. doi:10.1089/jwh.1999.8.399

59. Chavez NP, Voss A. Cáncer cérvico uterino e interculturalidad en Zona Maya, Quintana Roo, México. Tesis para optar por el grado de Maestría en Ciencias Sociales Aplicadas a los Estudios Regionales. Universidad de Quintana Roo. 2012. [Acceso 15 de junio de 2017]. Disponible en: http://www.uqroo.mx/dip/archivo/Tesis_Nancy_Chavez.pdf

60. Byrd TL, Peterson SK, Chavez R, Heckert A. Cervical cancer screening beliefs among young Hispanic women. Prevent Med 2004;38:192-7. 
61. Barrionuevo L. Desigualdades sociales en salud y el cribado de cáncer de cuello uterino y de mama en el Perú. (Tesis doctoral). 2015. [Acceso 15 de junio de 2017]. Disponible en: http://hdl.handle.net/10803/319716

62. Barrionuevo L, Palència L, Borrell C. ¿Cómo afecta el tipo de seguro de salud a la realización del Papanicolaou en Perú? Rev Panam Salud Publica 2013;34:393-400. [Acceso 15 de junio de 2017]. Disponible en: http://www.scielosp.org/scielo.php?script=sci_arttext\&pi $\mathrm{d}=\mathrm{S} 1020-49892013001200004$ 